\title{
Hyperforin induces apoptosis of chronic lymphocytic leukemia cells through upregulation of the BH3-only protein Noxa
}

\author{
MURHAF ZAHER ${ }^{1-3}$, RUOPING TANG ${ }^{1-3}$, ISABELLE BOMBARDA ${ }^{4}$, \\ FATEN MERHI $^{1-3}$, BRIGITTE BAUVOIS ${ }^{1-3}$ and CHRISTIAN BILLARD ${ }^{1-3}$ \\ ${ }^{1}$ Centre de Recherche des Cordeliers, INSERM U872, Equipe $18 ;{ }^{2}$ Université Pierre et Marie Curie; ${ }^{3}$ Université \\ Paris-Descartes, UMRS 872 Paris; ${ }^{4}$ ISM2-AD2M, UMR CNRS 6263, Université Paul Cézanne, Marseille, France
}

Received June 21, 2011; Accepted July 22, 2011

DOI: 10.3892/ijo.2011.1206

\begin{abstract}
We previously reported that hyperforin, a phloroglucinol purified from Hypericum perforatum, induces the mitochondrial pathway of caspase-dependent apoptosis in chronic lymphocytic leukemia (CLL) cells ex vivo, and that this effect is associated with upregulation of Noxa, a BH3-only protein of the Bcl-2 family. Here, we investigated the role of this upregulation in the pro-apoptotic activity of hyperforin in the cells of CLL patients and MEC-1 cell line. We found that the increase in Noxa expression is a time- and concentrationdependent effect of hyperforin occurring without change in Noxa mRNA levels. A post-translational regulation is suggested by the capacity of hyperforin to inhibit proteasome activity in CLL cells Noxa silencing by siRNA reduces partially hyperforin-elicited apoptosis. Furthermore, treatment with hyperforin, which has no effect on the expression of the prosurvival protein Mcl-1, induces the interaction of Noxa with Mcl-1 and the dissociation of Mcl-1/ Bak complex, revealing that upregulated Noxa displaces the proapoptotic protein Bak from Mcl-1. This effect is accompanied with Bak activation, known to allow the release of apoptogenic factors from mitochondria. Our data indicate that Noxa upregulation is one of the mechanisms by which hyperforin triggers CLL cell apoptosis. They also favor that new agents capable of mimicking specifically the $\mathrm{BH} 3$-only protein Noxa should be developed for apoptosis-based therapeutic strategy in CLL.
\end{abstract}

\section{Introduction}

In spite of recent therapeutic progresses, chronic lymphocytic leukemia (CLL) is still an incurable malignancy $(1,2)$. Because the leukemic B cells accumulating in the blood are mostly quiescent but defective in their cell death program, a

Correspondence to: Dr Christian Billard, UMRS 872, Equipe 18, Centre de Recherche des Cordeliers, 15 rue de l'Ecole de Médecine, 75006 Paris, France

E-mail: christian.billard@crc.jussieu.fr

Key words: hyperforin, CLL, apoptosis induction, Noxa upregulation, $\mathrm{Mcl}-1 / \mathrm{Bak}$ interaction new strategy of treatment based on the induction of apoptosis has attracted much attention $(3,4)$. Such a strategy requires the identification of specific targets. Mcl-1, a prosurvival member of the Bcl-2 family (5) which is overexpressed by CLL cells (6), appears as a target of choice for therapeutic purpose. Actually, this protein is now thought to play a role of prime importance in the apoptotic deficiency (7). In particular, its expression is correlated with prognostic markers and clinical outcomes of CLL patients $(8,9)$, and its loss is sufficient to trigger in vitro apoptosis (10). Mcl-1 can be down-regulated by flavopiridol, a semi-synthetic flavonoid of vegetal origin known to be a potent inducer of CLL cell apoptosis in vitro through its property to inhibit short-lived protein mRNA transcription (11). However, this effect is not specific and flavopiridol has not been approved by FDA. The BH3-only protein Noxa seems also to be a potential therapeutic target for CLL because it is a specific ligand of Mcl-1 which neutralizes its anti-apoptotic activity (12). In addition, Noxa expression is inversely related to CLL cell survival capacity (13) and its enhancement is involved in the proapoptotic effects of proteasome or histone deacetylase (HDAC) inhibitors on CLL cells $(13,14)$. Nevertheless, both types of agents also enhance Mcl-1 expression $(15,16)$, and it is noteworthy that their use in clinical trials on CLL were disappointing $(17,18)$.

Hyperforin is a plant-derived inducer of CLL cell apoptosis that we have previously described (19) and which seems of particular interest for its pleiotropic properties. This phloroglucinol purified from Hypericum perforatum (St John's wort) has been characterized as the main active component of this plant used for centuries to treat mild depression. Hyperforin displays antibacterial, antioxidant and immunomodulating activities, inhibits inflammation and allergy mediators, has antitumor, anti-angiogenic and antimigratory effects in vitro and in animal models (20). In CLL cells, hyperforin is capable of inhibiting the production of two mediators of angiogenesis, metalloproteinase-9 and VEGF and the activity of proteins involved in multidrug resistance, as reported by us $(21,22)$. Like most CLL cell apoptosis inducers, hyperforin triggers the caspasedependent mitochondrial pathway (19) but the molecular mechanisms allowing the liberation of apoptogenic factors from mitochondria are not elucidated. In a preliminary study, we found that Noxa protein is upregulated during apoptosis of CLL cells induced by hyperforin but not flavopiridol (23). 
In the present study, we first characterized this Noxa upregulation and then provided evidence favoring that Noxa is involved in the mechanism of action of the phloroglucinol through interaction with Mcl-1, displacement of the proapoptotic protein Bak from its complex with Mcl-1 and Bak activation, responsible for mitochondrial membrane permeabilization. Our data thus strengthen the concept of targeting the BH3-only protein Noxa for CLL therapy.

\section{Patients and methods}

Patients, cells and cell culture. Blood samples from CLL patients were obtained from the Hematology Department of SaintAntoine Hospital (Paris, France) after written informed consent in accordance with the revised Helsinki protocol. Diagnosis was established according to the International Workshop on CLL (IWCLL), including lymphocyte morphology and co-expression of CD5, CD20 and CD23 antigens (24). A total of 22 untreated patients (16 men and 6 women), all classified as Binet's stage A, with a mean age of $64 \pm 13$ years, were selected. Leukemic cells from CLL patients were purified as previously reported to a purity $\geq 95 \%$ (25) and were immediately cultured. Cell cultures were performed at $37^{\circ} \mathrm{C}$ in a humidified atmosphere containing $5 \% \mathrm{CO}_{2}$ in RPMI-1640 medium supplemented with $2 \mathrm{mM}$ glutamine, antibiotics and 10\% FCS (PAA Laboratories, Pasching, Austria) at seeding densities of $2-4 \times 10^{6}$ cells $/ \mathrm{ml}$. The MEC-1 cell line, established from a CLL patient in prolymphocytoid transformation (26) was purchased from DSMZ (Braunschweig, Germany). MEC-1 cells were routinely cultured between $2 \times 10^{5}$ and $10^{6}$ cells $/ \mathrm{ml}$ in the same medium as above but supplemented with $1 \mathrm{mM}$ sodium pyruvate, and experiments were carried out by seeding cells at $5 \times 10^{5} / \mathrm{ml}$. Cell concentrations were measured with a Z-2 Coulter counter (Beckman-Coulter, Les Ulis, France) and cell viability was evaluated using a colorimetric assay based on the cleavage of the tetrazolium salt WST-1 by mitochondrial dehydrogenases (Roche Diagnostics, Meylan, France).

Reagents. Hyperforin (MW 538) was purified by one of us (IB) in the laboratory which described the procedure (27). The protocol uses alkaline medium in order to exclude hypericin due to its different solubility properties and to insure the stability of the product during the purification $(\mathrm{pH}$ control, solvent nature, evaporation). The purity of the preparation was $>98 \%$ as monitored by reversed-phase HPLC-DAD technique with $<1 \%$ hypericin. Hyperforin was stored frozen in ethanolic solution under conditions preventing its sensitivity to light, oxygen and aqueous solvent to avoid oxidation products. The biological activity of our preparation was similar to that of commercial hyperforin (purity $>90 \%$, Cayman Chemical, Interchim, Montlucon, France) as assessed by apoptosis induction assay with MEC-1 and U937 cell lines. Flavopiridol was kindly provided by Aventis Pharmaceuticals (Bridgewater, NJ, USA). Other reagents and chemicals were from Sigma (Sigma Aldrich, Saint Quentin Fallavier, France), except when otherwise stated.

Western blotting. Protein expression was studied by Western blotting from cell lysates containing equal amounts of proteins $(30-50 \mu \mathrm{g})$, as detailed previously (25). Proteins were revealed by blotting with the following primary antibodies: the rabbit polyclonal (S-19) anti-Mcl-1 (from Santa Cruz Biotechnology, Tebu, Le Perray en Yvelines, France); two mouse mAb antiNoxa, clone 6D619 (US Biologicals, Euromedex, Mundolsheim, France) and IMG-249 (Imgenex, Clinisciences, Montrouge, France); the mouse mAb anti-Bak Ab-1 specific for the active form of Bak (Calbiochem, VWR International SAS, Fontenay, France); an anti-PARP-1 [poly(ADP-ribose) polymerase-1] recognizing both the native form $(116 \mathrm{kDa})$ and the cleaved fragment (85 kDa) from Santa Cruz Biotechnology; an anti- $\beta$-actin (clone C4; MP Biomedicals, Illkirch, France) used for protein content monitoring and standardization. Band intensities of the proteins visualized in Western blots were quantified with the NIH Image 1.62b software.

Immunoprecipitation experiments. Cells were lysed for $30 \mathrm{~min}$ at $4{ }^{\circ} \mathrm{C}$ with $1 \% 3$-[(3-cholamidopropyl)dimethylammonio]1-propane sulfonate (CHAPS), $20 \mathrm{mM}$ Tris- $\mathrm{HCl} / \mathrm{pH} \mathrm{8.0,}$ $150 \mathrm{mM} \mathrm{NaCl}$ and a cocktail of protease inhibitors. Cell lysates were pre-cleared for $30 \mathrm{~min}$ at $4^{\circ} \mathrm{C}$ with Protein G-agarose beads (Santa Cruz Biotechnology) equilibrated with lysis buffer in the presence of isotype antibody. Pre-cleared lysates were then incubated overnight at $4^{\circ} \mathrm{C}$ with pre-equilibrated Protein $\mathrm{G}$ beads and the relevant antibody: the mouse $\mathrm{mAb}$ anti-Mcl-1 (MCL-1801 from Santa Cruz Biotechnology) or the rabbit polyclonal anti-Bak NT (Upstate, Millipore, Saint Quentin en Yvelines, France). Immunoprecipitates (IP) were washed and mixed with non-reducing electrophoresis sample buffer, and beads were discarded after boiling for $3 \mathrm{~min}$. IP were finally subjected to Western blot analysis using either antiMcl-1 (S-19), anti-Noxa (6D619) or anti-Bak (NT) primary antibodies.

Proteasome activity assay. Proteasome activity was determined with the aid of the Proteasome-Glo ${ }^{\mathrm{TM}}$ Chymotrypsin-like cellbased assay (Promega France, Charbonnières-les-Bains, France) that measures chymotrypsin-like protease activity associated with the proteasome complex directly in intact cells. This assay has been used to evaluate the activity of bortezomib in a variety of cancer cell lines (28). It has three components: a buffer containing the proteasome complex, a luciferin detection reagent and the luminogenic substrate, succinyl-LLVY-aminoluciferin. The proteasome cleavage generates a luminescent signal produced by luciferase reaction. Briefly, $100 \mu \mathrm{l}$ of the reagent mixture was added to $2 \times 10^{5}$ cells $/ 100 \mu \mathrm{l}$ in 96 -well micro-titration plates for $15 \mathrm{~min}$ at $37^{\circ} \mathrm{C}$, and the luminescence was immediately recorded

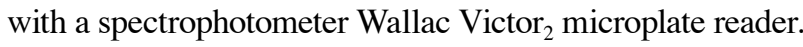

RT-PCR analysis of mRNA expression. Total RNA was extracted from CLL cells with the Trizol method and reverse transcription was performed as previously detailed (29). The primers used for Noxa and Mcl-1 (synthesized by Eurobio, Les Ulis, France) and for $\beta 2$-microglobulin (Sigma Proligo France) were as follows: Noxa (forward) 5'-GTG CCC TTG GAA ACG GAA GA-3', and (reverse) 5'-CCA GCC GCC CAG TCT AAT CA-3'; Mcl-1 (forward) 5'-ATC TCT CGG TAC CTT CGG GAG C-3', and (reverse) 5'-CCT GAT GCC ACC TTC TAG GTC C-3'; $\beta 2$-microglobulin (forward) 5'-CAT CCA GCG TAC TCC AAA GA-3', and (reverse) 5'-GAC AAG TCT GAA TGC TCC A-3'. PCR products were visualized by electrophoresis in $2 \%$ agarose gel containing $0.2 \mathrm{mg} / \mathrm{ml}$ ethidium bromide. The 
A

\begin{tabular}{|c|c|c|c|c|c|c|c|c|c|}
\hline & \multicolumn{3}{|c|}{ CLL\# 1} & \multicolumn{3}{|c|}{ CLL\# 15} & \multicolumn{3}{|c|}{ MEC-1 } \\
\hline & C & $\mathbf{F}$ & $\mathbf{H}$ & $\mathrm{C}$ & $\mathbf{F}$ & $\mathbf{H}$ & C & $\mathbf{F}$ & $\mathbf{H}$ \\
\hline $\begin{array}{r}\text { PARP native } \\
\text { cleaved }\end{array}$ & & & $=$ & - & & - & - & & $=$ \\
\hline Noxa & - & & - & 5 & & $\sim$ & - & 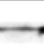 & $\sigma$ \\
\hline Mcl-1 & - & $\longrightarrow$ & - & tert & & $\rightarrow$ & - & - & \\
\hline Actin & 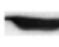 & & & & & & & & \\
\hline
\end{tabular}

\section{B}

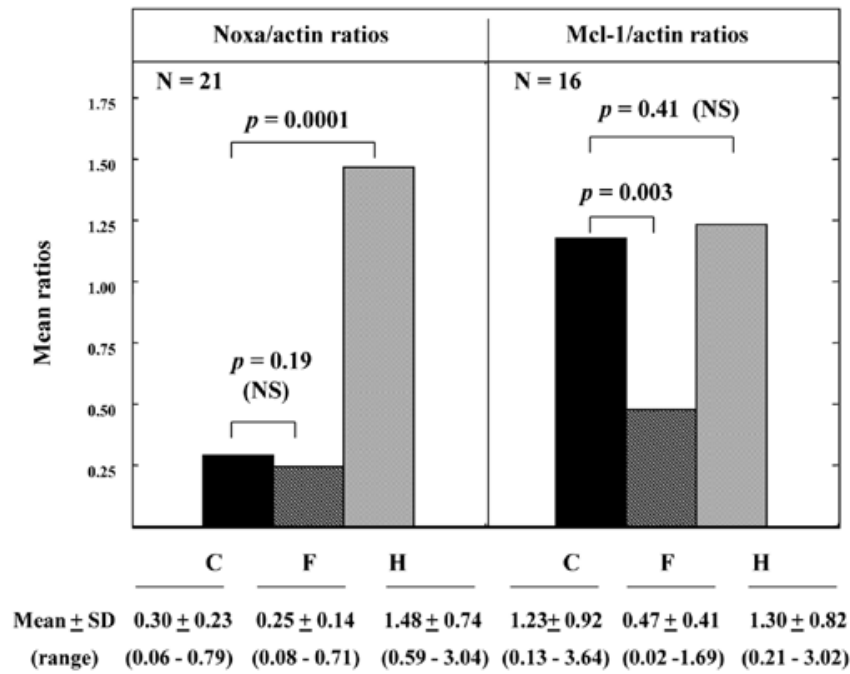

C

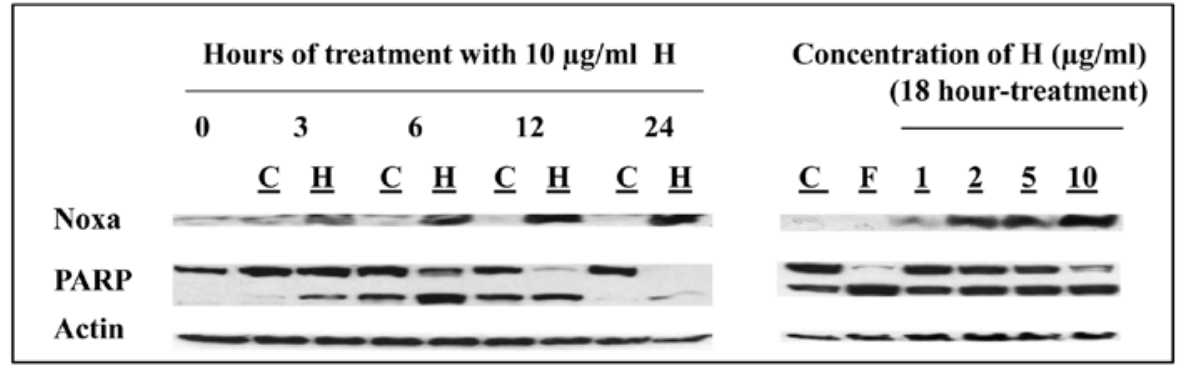

Figure 1. Effect of hyperforin on Noxa protein expression in CLL cells and comparison with flavopiridol. Cells from 21 patients were treated for $18 \mathrm{~h}$ with $10 \mu \mathrm{g} / \mathrm{ml}$ $(18 \mu \mathrm{M})$ hyperforin $(\mathrm{H})$ or $1 \mu \mathrm{M}$ flavopiridol (F) or left untreated (controls, C) before Western blot analysis. (A) Hyperforin induces Noxa up-regulation associated with PARP-1 cleavage (an index of apoptosis induction) as exemplified with two patients; the right panel shows a representative experiment with MEC-1 cells (out of three that gave similar results, after treatment with $10 \mu \mathrm{g} / \mathrm{ml} \mathrm{H}$ but $0.2 \mu \mathrm{M} \mathrm{F}$ ). (B) Global analysis of the effects of $\mathrm{H}$ and $\mathrm{F}$ on Noxa/actin band intensity ratios in CLL cells (patients nos. 1-19,21 and 22) and on Mcl-1/actin ratios (patients nos. 1-10, 15-19 and 21), with p-values evaluated using the unpaired Student's t-test; NS, not significant. (C) Noxa upregulation depends on time and concentration of treatment with hyperforin in CLL cells. Data are from one experiment representative of two other experiments (patients nos. 19, 20 and 22).

sizes of amplified cDNAs were $165 \mathrm{bp}$ for $\beta 2$-microglobulin, $446 \mathrm{bp}$ for Mcl-1, and $260 \mathrm{bp}$ for Noxa. Band intensities were quantified by the NIH Image 1.63 software after acquisition in an Appligen densitometer (Oncor).

siRNA nucleofection. Experiments of mRNA silencing were carried out by transfecting cells with small interfering RNA (siRNA). The Noxa siRNA and negative control siRNA were purchased from Qiagen (Courtaboeuf, France). Nucleofection of cells was performed using the Amaxa Human B cell
Nucleofector kit (Lonza, Levallois-Perret, France) and the Nucleofector System of Amaxa (Cologne, Germany). Briefly, CLL $\left(2 \times 10^{6}\right)$ or MEC-1 $\left(10^{6}\right)$ cells were mixed with either Noxa or control siRNA (30 $\mathrm{nM})$ in the buffer of the kit and immediately nucleofected using different programs according to the manufacturer's instructions. Cells were immediately transferred in culture plates containing complete medium (final volume of $1 \mathrm{ml}$ ) and incubated at $37^{\circ} \mathrm{C}$ for $5 \mathrm{~h}$ to allow siRNA transfection. Then, transfected cells were either treated with different concentrations of hyperforin or left untreated for $42 \mathrm{~h}$ prior to measure 
phosphatidylserine externalization. The effects of hyperforin were checked in parallel on cells which were not transfected as a control experiment.

Phosphatidylserine (PS) externalization evaluation. PS externalization, a membrane marker of apoptosis was quantified by specific binding of FITC-conjugated annexin V (Bender Medsystems, Vienna, Austria) with or without simultaneous labeling with propidium iodide (PI), as previously detailed (25). The percentages of cells positive for annexin V-FITC/PI negative (early apoptosis) and of double positive cells (late apoptosis) were determined with a flow cytometer (Beckman Coulter, Luminy, France). Results are expressed as the percentages of total annexin $\mathrm{V}$ positive cells.

\section{Results}

Hyperforin induces a concentration and time-dependent Noxa upregulation. As previously shown (19), the maximal effects of hyperforin in inducing apoptosis of primary CLL cells in culture (DNA fragmentation, PS externalization, mitochondrial membrane depolarization, caspase-3 activation, PARP-1 cleavage) are achieved by $18-24 \mathrm{~h}$ of treatment with $10 \mu \mathrm{g} / \mathrm{ml}$ $(18 \mu \mathrm{M})$. These experimental conditions were used to investigate the effects of hyperforin on Noxa protein expression in CLL cells from a total of 21 patients. Under these conditions, no change in cell viability was detected, cell mortality occurring after at least $48 \mathrm{~h}$ of treatment. As illustrated in Fig. 1A, low or hardly detectable levels of Noxa expression in untreated cells increased to high levels in hyperforin-treated cells. This effect was observed in all 21 patients and was always associated with caspase-dependent PARP-1 cleavage, a control of apoptosis induction. However, no augmentation of Noxa expression was detected upon treatment with flavopiridol which, as expected, markedly decreased Mcl-1 levels (Fig. 1A). These results, which were in accordance with the preliminary data (23), were similarly observed with the cell line MEC-1 (Fig. 1A). The mean ratios of Noxa/actin and Mcl-1/actin band intensities obtained with all the patients tested show that hyperforin-induced Noxa upregulation (5-fold increase versus untreated controls) was highly significant, while Mcl-1 levels did not vary significantly (Fig. 1B). Western blots with two different anti-Noxa antibodies provided the same results, which were also found by immunofluorescence and flow cytometry analysis (data not shown). The increase in Noxa levels depended on hyperforin concentration and time of treatment in CLL cells from 3 patients and MEC-1 cells, peaking between 12 and $24 \mathrm{~h}$ with $10 \mu \mathrm{g} / \mathrm{ml}$ of hyperforin, as exemplified in Fig. 1C. Therefore, Noxa upregulation is actually an effect of hyperforin which is associated with apoptosis induction in CLL cells and occurs without significant modulation of Mcl-1 expression.

Hyperforin has no effect on Noxa mRNA expression. The effects of hyperforin on Noxa mRNA expression were studied by semi-quantitative RT-PCR. Cells were cultured in the presence of various hyperforin concentrations and for different times under experimental conditions allowing apoptosis induction and Noxa protein up-regulation. The results which were similar for CLL cells from three patients and MEC-1 cells are exemplified in Fig. 2A-C. They revealed no change in Noxa mRNA levels versus untreated controls within $24 \mathrm{~h}$ of treatment with hyperforin concentrations $\geq 12 \mu \mathrm{g} / \mathrm{ml}$, while these levels were either or not diminished upon exposure to flavopiridol. The paralleled analysis of Mcl-1 mRNA expression showed no effect of hyperforin but, as expected, a marked decrease after flavopiridol treatment (Fig. 2A-C). These data suggest that the effect of hyperforin on Noxa protein levels does not result from transcriptional activation.

Hyperforin inhibits proteasome activity. The inhibition of nonlysosomal proteolysis of ubiquitinated proteins by the proteasome is known to cause increased expression of certain proteins (30). Because Noxa can be upregulated by the proteasome inhibitor bortezomib in various cell types including CLL cells $(13,30)$ and because a number of natural compounds are proteasome inhibitors $(31,32)$, we therefore investigated the capacity of hyperforin to inhibit proteasomal activity in CLL cells in comparison with bortezomib as a reference. Fig. 2D shows that hyperforin treatment resulted in concentration-dependent reduction of proteasome activity, although to an extent lesser than the inhibition by bortezomib. The maximal effects were reached with elevated concentrations of hyperforin $(15-20 \mu \mathrm{g} / \mathrm{ml})$, i.e., concentrations higher than those inducing optimal Noxa upregulation and apoptosis $(10 \mu \mathrm{g} / \mathrm{ml})$, and comparable results were obtained with MEC-1 cell line (Fig. 2D). The data indicate that hyperforin is capable of inhibiting proteasomal degradation in CLL cells, supporting the assumption that the phloroglucinol might enhance Noxa expression through a post-translational regulation at the protein level.

Noxa silencing reduces hyperforin-induced apoptosis. The effect of Noxa silencing on hyperforin-elicited apoptosis was investigated. Cells were transfected with Noxa siRNA or a negative control siRNA, then treated with hyperforin or left untreated prior to measurement of PS externalization. Results obtained in two experiments with MEC-1 cells and in CLL patient's cells are exemplified in Fig. 3A. In the absence of hyperforin treatment, the levels of PS externalization were similar after nucleofection with either negative control siRNA or Noxa siRNA (while higher than in untransfected cells, not shown). Upon hyperforin exposure, the percentage of cells positive for PS externalization markedly increased in cells which have been transfected with negative control siRNA (to levels similar to those of untransfected cells). These increases were reduced when cells were transfected with Noxa siRNA by 46 and 23\% in the experiments with MEC-1 cells and by $32 \%$ in CLL cells (Fig. 3A). The effect of Noxa siRNA was reproducibly observed and its magnitude was comparable to that was previously found with various cell types including CLL cells (13,14,33-35). Consequently, loss of Noxa expression reduces partially hyperforin-induced apoptosis, indicating that the $\mathrm{BH} 3$-only protein mediates in part this apoptosis induction and that at least another mechanism is involved.

Hyperforin treatment induces Noxa/Mcl-1 association and Mcl-1/Bakdissociation. Experiments of co-immunoprecipitation were performed with cells committed to apoptosis by hyperforin treatment and with untreated controls in order to examine the effects of the phloroglucinol on Noxa/Mcl-1 and Mcl-1/Bak interactions (Fig. 3B and C). Exposure of CLL patient cells to hyperforin but not flavopiridol strongly induced the co-preci- 


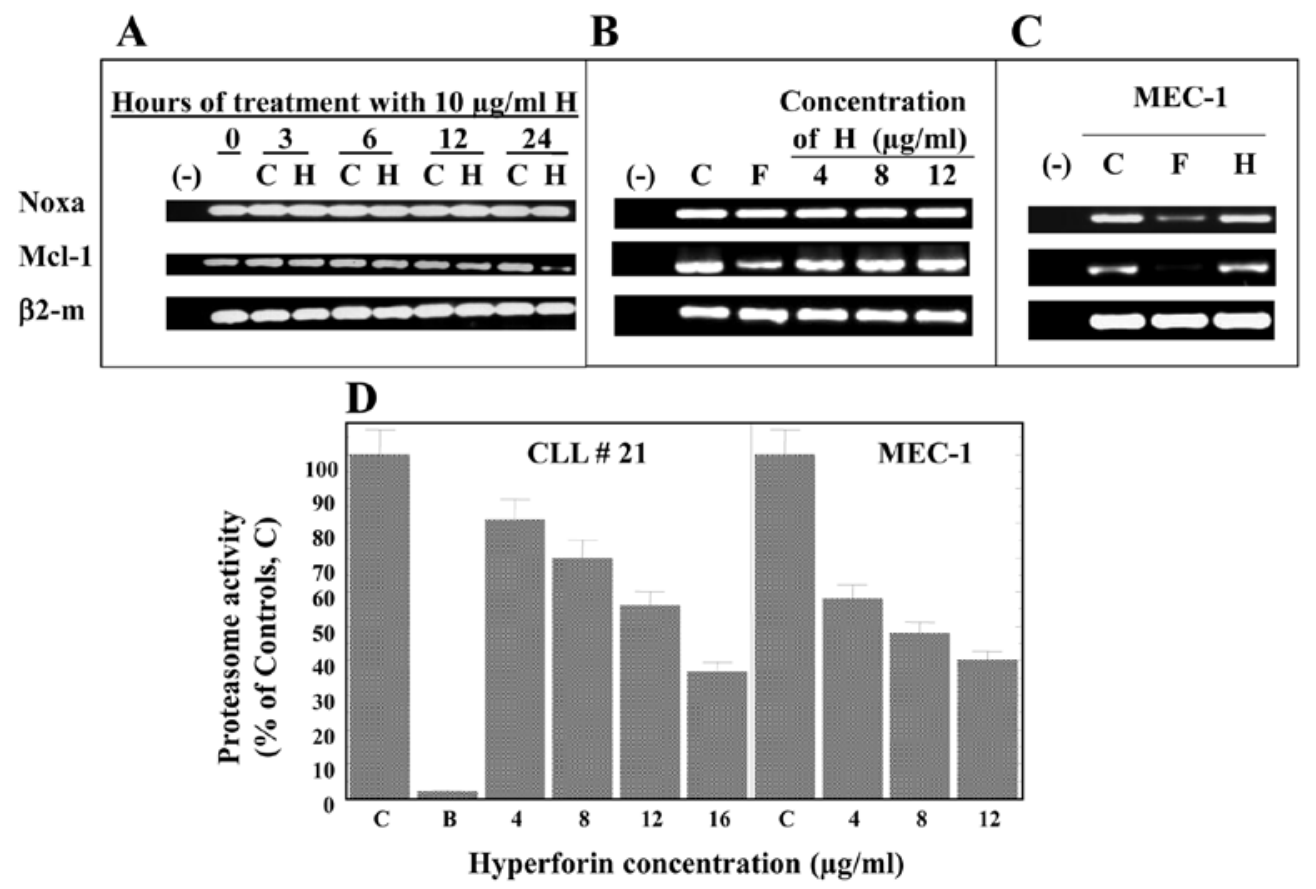

Figure 2. Hyperforin does not change Noxa mRNA expression but inhibits proteasome activity in CLL and MEC-1 cells. Cells from 3 patients (nos. 17, 18 and 21) and MEC-1 cells were treated for different times with various concentrations of hyperforin (H) or left untreated (controls, C). (A) Effect of time of treatment with hyperforin on Noxa mRNA and Mcl-1 mRNA levels in CLL cells from patient no. 21, as analyzed by RT-PCR using $\beta 2$-microglobulin ( $\beta 2$-m) for RNA content monitoring. (B) Effect of hyperforin concentration on Noxa and Mcl-1 mRNA levels in cells from CLL no. 21 (18-h treatment); the effect of $1 \mu \mathrm{M}$ flavopiridol was examined in parallel. (C) RT-PCR analysis after treatment of MEC-1 cells with $10 \mu \mathrm{g} / \mathrm{ml} \mathrm{H}$ or $0.2 \mu \mathrm{M}$ flavopiridol (F) for $18 \mathrm{~h}$. (-), Buffer without cDNA. (D) Dose-dependent inhibition of proteasomal degradation by hyperforin in CLL no. 21 and MEC-1 cells after treatment for $18 \mathrm{~h}$ with increasing concentrations of hyperforin, or with $10 \mathrm{nM}$ bortezomib (B) as a proteasome inhibitor. For technical procedures, see Materials and methods. Data exemplified with patient no. 21 were similar with the two other patients.

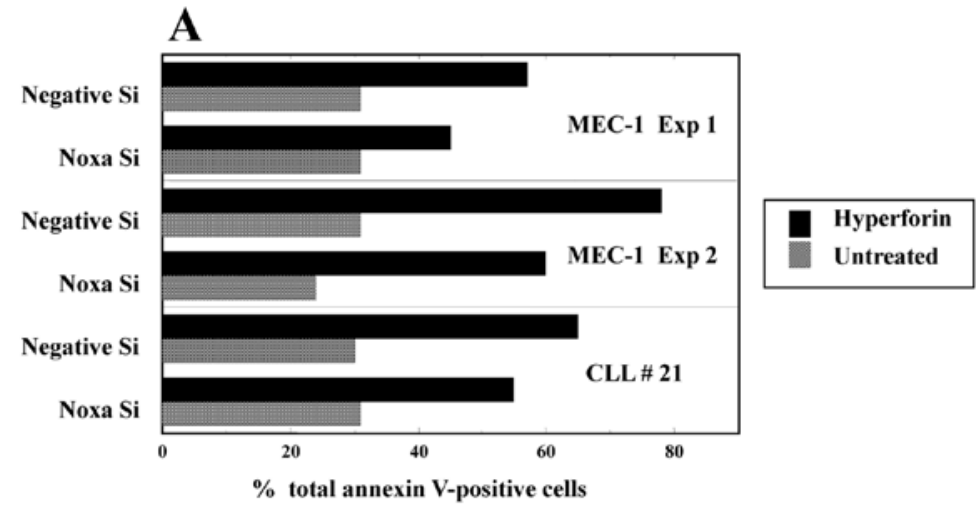

B

C

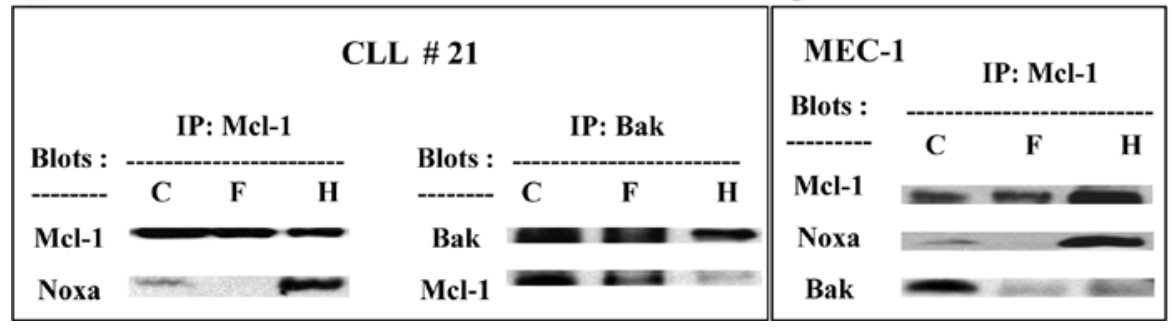

Figure 3. Noxa is involved in hyperforin-triggered apoptosis of primary CLL cells (as illustrated with patient no. 21) and MEC-1 cells through interaction with Mcl-1 and dissociation of Mcl-1/Bak complex. (A) Noxa silencing reduces PS externalization induced by hyperforin treatment: cells were transfected with either Noxa siRNA (Noxa Si) or negative control siRNA (negative Si), then treated with $5 \mu \mathrm{g} / \mathrm{ml}$ hyperforin or left untreated for $42 \mathrm{~h}$ before measurement of PS externalization by annexin V fixation. (B) Hyperforin both induces the co-precipitation of Noxa with Mcl-1 and abrogates the co-precipitation of Mcl-1 with Bak in CLL cells. Cells treated for $18 \mathrm{~h}$ with $10 \mu \mathrm{g} / \mathrm{ml}$ hyperforin $(\mathrm{H})$ or $1 \mu \mathrm{M}$ flavopiridol (F) and untreated controls $(\mathrm{C})$ were lysed, and the lysates were simultaneously immunoprecipitated with either an anti-Mcl-1 or an anti-Bak. Mcl-1 immunoprecipitates (IP) were immunoblotted with anti-Mcl-1 (IP control) or anti-Noxa to evaluate Mcl-1/Noxa interaction. Bak IP were blotted with anti-Bak (IP control) or anti-Mcl-1 to examine Mcl-1/Bak association. The total levels of Noxa, Mcl-1 and Bak were in accordance with Fig. 1 and Table I. Similar results were obtained with three other patients (nos. 19, 20 and 22). (C) Co-precipitation experiments performed with MEC-1 cell lysates. 
Table I. Effects of hyperforin on the expression of the activated form of Bak in CLL cells and comparison with the effect of flavopiridol.

\begin{tabular}{llll}
\hline & \multicolumn{2}{c}{ Active Bak/ $\beta$-actin ratios $(\mathrm{N}=8$ CLL patients) } \\
\cline { 2 - 4 } & Controls & Flavopiridol & Hyperforin \\
\hline Mean \pm SE & $0.45 \pm 0.05$ & $2.39 \pm 0.97$ & $1.55 \pm 0.69$ \\
(range) & $(0.27-0.71)$ & $(0.33-8.98)$ & $(0.56-6.28)$ \\
t-test & & $\mathrm{P}=0.02$ & $\mathrm{P}=0.01$ \\
\hline
\end{tabular}

CLL cells from 8 patients (nos. 13-15, 17-19, 21 and 22) were treated for $18 \mathrm{~h}$ with $10 \mu \mathrm{g} / \mathrm{ml}$ hyperforin or $1 \mu \mathrm{M}$ flavopiridol or left untreated (controls) and then analyzed by Western blotting with an antibody specific for the active form of Bak. The ratios of active Bak/ $/ \beta$-actin band intensities were evaluated as described in Materials and methods and are expressed as means \pm standard error (SE). P-values were calculated using the unpaired Student's t-test.

pitation of Noxa with Mcl-1 as compared to untreated controls (Fig. 3B). Simultaneously, hyperforin treatment abolished the co-precipitation of Bak with Mcl-1 detected in untreated controls (Fig. 3B). These effects, which were always associated with Noxa upregulation, were similarly observed with the MEC-1 cell line (Fig. 3C). The data clearly demonstrate that hyperforin induces both the interaction of Noxa with Mcl-1 and the dissociation of Mcl-1/Bak complex, indicating that Noxa displaces Bak from Mcl-1. As expected, Mcl-1/Bak association was also reduced upon treatment with flavopiridol (Fig. 3B and C) which consistently decreased Mcl-1 levels (Fig. 1A and B).

Hyperforin treatment increases activated Bak expression. To verify if hyperforin-induced displacement of Bak from its complex with Mcl-1 might result in Bak activation, Western blot experiments were performed with an antibody recognizing specifically the active form of Bak. Table I shows that hyperforin treatment increased significantly the expression of activated Bak in CLL cells from 8 patients. Similarly, this effect was observed upon flavopiridol treatment which also decreased the levels of interaction of Mcl-1 with Bak (Fig. 3B and C). The augmentation of active Bak levels by hyperforin was associated with Noxa upregulation and PARP-1 cleavage as found in cells of three patients (data not shown). The data thus indicate that hyperforin-elicited Noxa upregulation and Mcl-1/Bak disruption are accompanied with Bak activation.

\section{Discussion}

The characterization of the Noxa upregulation associated with hyperforin-induced apoptosis of CLL cells reveals that it is a concentration and time-dependent effect of the phloroglucinol occurring in CLL cells from all the patients in the study. This effect does not seem to result from transcription activation but rather from a regulation at the protein level, as suggested by the capacity of hyperforin to inhibit proteasome activity. These data are not surprising since it is known that the BH3-only protein Noxa can be activated by either transcriptional or post-translational regulation depending on the experimental systems (36). In particular, Noxa induction is p53-independent in $\mathrm{B}$ cell malignancies and moreover, inhibition of proteasomal degradation causes Noxa stabilization and accumulation in CLL cells notably $(13,33)$. In addition, a number of plant-derived compounds are natural proteasome inhibitors $(31,32)$. While the mechanism involved and a statistical analysis with a large number of patients are under study, we can claim that the inhibitory effect of hyperforin on proteasomal activity is not due to the contamination of our hyperforin preparation by hypericin, one of the major constituents of St John's wort which is known as a proteasome inhibitor, because of the high level of purity of this preparation (see Materials and methods). Furthermore, loss of Noxa by RNA silencing consistently reduces hyperforintriggered apoptosis in both MEC-1 and primary CLL cells. Although the MEC-1 cell line was established from a CLL patient in prolymphocytic transformation (26) and thus, is not stricto sensus a CLL cell line, our observations throughout the study that MEC-1 cells behave quite similarly to CLL patient cells regarding their responses to hyperforin indicate that this cell line constitutes a relevant model to study the mechanism of action of the phloroglucinol. The fact that Noxa silencing reduces only partially hyperforin-promoted apoptosis suggests the involvement of at least another mechanism. Finally, we describe three molecular events occurring as a consequence of hyperforin-elicited Noxa upregulation in CLL cells: i) Noxa interacts with Mcl-1, ii) Bak is displaced from its complex with Mcl-1, as already found upon Noxa induction by bortezomib and HDAC inhibitors $(13,14)$, and iii) Bak is activated. These three steps are known to cause mitochondrial membrane permeabilization and subsequent caspase cascade $(5,12)$.

Together, data of the present study indicate that Noxa upregulation is one of the mechanisms through which hyperforin triggers the caspase-dependent mitochondrial apoptosis in CLL cells. Considering other possible mechanisms, the phloroglucinol might also enhance BH3-only proteins such as Bim or Puma which are known to neutralize the prosurvival Bcl-2 family members Bcl-xL, Bcl-w or Bcl-2 itself. In connection with this, Puma gene expression was reported to be activated during CLL cell apoptosis promoted by fludarabine (37), Bim was involved in the effects of HDAC inhibitors or glucocorticoids $(14,38)$, and the role of Bcl-xL in CLL has been highlighted $(39,40)$. Experiments are in progress to study the effects of hyperforin on these various Bcl-2 family members, as well as on Bax, the proapoptotic ligand of Bcl-xL allowing mitochondrial membrane permeabilization. Furthermore, hyperforin might stimulate another pathway of apoptosis such as caspase-independent programmed cell death (also associated with PS externalization) which has been shown to play a role in CLL cells (41). Finally, we cannot exclude that the inhibitory properties of hyperforin on sirtuins, which are HDAC capable of preventing apoptosis (42), may be implicated.

The new steps in the action of hyperforin described in the present work reveal that this apoptosis inducer uses a mechanism different from that of flavopiridol to target the same prosurvival protein Mcl-1: while the flavonoid down-regulates Mcl-1 through non-specific transcription inhibition of short-lived proteins (11), the phloroglucinol specifically neutralizes Mcl-1 anti-apoptotic activity by upregulating Noxa; both mechanisms result in Bak activation. Consequently, hyperforin seemingly acts in a way resembling that of proteasome inhibitors. Nevertheless, unlike 
proteasome inhibitors and HDAC inhibitors as well $(15,16)$, hyperforin does not enhance Mcl-1 expression within the range of concentrations provoking optimal Noxa up-regulation and apoptosis in CLL cells. Only slight increases in Mcl-1 levels were occasionally detected in some patients after treatment with high hyperforin concentrations corresponding to maximal inhibition of proteasomal activity (15-20 $\mu \mathrm{g} / \mathrm{ml})$. Decreases in Mcl-1 levels were even observed, being caspase-dependent and thus indirect consequences of apoptosis induction (19). Such variations in Mcl-1 levels might reflect differences in leukemic cell sensitivity to hyperforin between CLL patients. Experiments are in progress to elucidate whether hyperforin inhibits Noxa degradation via a mechanism similar to bortezomib or other proteasome inhibitors (33).

When considering the relevance of hyperforin for clinical application, several properties of hyperforin that limit its use have to be mentioned: modulation of cytochrome P450 and interference with the clearance of xenobiotics (20) or sensitivity of the phloroglucinol to light, oxygen and aqueous solvent (43). However, new chemical derivatives have been developed with improved stability and solubility and more potent activity, such as aristoforin (43). Obviously, preclinical experiments on the sensitivity of bone marrow progenitors and normal $\mathrm{B}$ and $\mathrm{T}$ lymphocytes and on the co-incubation with microenvironment cells protecting leukemic cells from apoptosis (3) should be performed. To evaluate the efficacy of hyperforin derivatives in CLL cells from p53 mutated patients might also enhance their clinical relevance (2).

Finally, since the emergence of the concept that agents capable of mimicking $\mathrm{BH} 3$-only proteins may be of interest for therapeutic strategies based on apoptosis induction $(5,12)$, several pan-BH3 mimetics were studied with somewhat promising results $(39,44,45)$. Our present study prompts the development of new BH3 mimetics specific for Noxa which seem crucial for improving CLL therapy.

\section{Acknowledgements}

We are indebted to Professor Jean-Pierre Marie for providing patient blood samples. We thank Mrs. Fanny Fava, Julia Prado and Solenne Biston for their expert technical assistance.

\section{References}

1. Tam CS and Keating MJ: Chemoimmunotherapy of chronic lymphocytic leukemia. Nat Rev Clin Oncol 7: 521-532, 2010.

2. Dighiero G and Hamblin DJ: Chronic lymphocytic leukemia. Lancet 371: 1017-1029, 2008.

3. Pleyer L, Egle A, Hartmann TN and Greil R: Molecular and cellular mechanisms of CLL: novel therapeutic approaches. Nat Rev Clin Oncol 6: 405-418, 2009.

4. Kolb JP,Kern C, Quiney C, Roman V and BillardC:Re-establishment of a normal apoptotic process as a therapeutic approach in B-CLL. Curr Drug Targets Cardiovasc Hematol Disord 3: 261-286, 2003.

5. Adams JM and Cory S: The Bcl-2 apoptotic switch in cancer development and therapy. Oncogene 26: 1324-1337, 2007.

6. Kitada S, Andersen J, Akar S, et al: Expression of apoptosisregulating proteins in chronic lymphocytic leukemia: correlations with in vitro and in vivo chemoresponses. Blood 91: 3379-3389, 1998.

7. Gandhi V, Balakrishnan K and Chen LS: Mcl-1: the 1 in CLL. Blood 112: 3538-3540, 2008.

8. Pepper C, Lin TT, Pratt G, et al: Mcl-1 expression has in vitro and in vivo significance in chronic lymphocytic leukemia and is associated with other poor prognostic markers. Blood 112: 3807-3817, 2008 .
9. Awan FT, Kay NE, Davies ME, et al: Mcl-1 expression predicts progression-free survival in chronic lymphocytic leukemia patients treated with pentostatin, cyclophosphomide, and rituximab. Blood 133: 535-537, 2009.

10. Hussain SR, Cheney CM, Johnson AJ, et al: Mcl-1 is a relevant therapeutic target in acute and chronic lymphoid malignancies: downregulation enhances rituximab-mediated apoptosis and complement-dependent cytotoxicity. Clin Cancer Res 13: 144-150, 2007.

11. Chen R, Keating MJ, Gandhi V and Plunkett W: Transcription inhibition by flavopiridol: mechanism of chronic lymphocytic leukemia cell death. Blood 106: 2513-2519, 2005.

12. Willis SN, Chen L, Dewson G, et al: Proapoptotic Bak is sequestered by Mcl-1 and Bcl-xL, but not Bcl-2, until displaced by BH3-only proteins. Genes Dev 19: 1294-1305, 2005.

13. Smit L, Hallaert DYH, Spijker R, et al: Differential Noxa/Mcl-1 balance in peripheral versus lymph node chronic lymphocytic leukemia cells correlates with survival capacity. Blood 109: 1660-1668, 2007.

14. Inoue S, Riley J Gant TW, Dyer MJS and Cohen GM: Apoptosis induced by histone deacetylase inhibitors in leukemic cells is mediated by Bim and Noxa. Leukemia 21: 1773-1782, 2007.

15. Nencioni A, Hua F, Dillon CP, et al: Evidence for a protective role of Mcl-1 in proteasome inhibitor-induced apoptosis. Blood 105: 3255-3262, 2005.

16. Inoue S, Walewska R, Dyer MJS and Cohen GM: Downregulation of Mcl-1 potentiates HDACi-mediated apoptosis in leukemic cells. Leukemia 22: 819-825, 2008.

17. Faderl S, Rai K, Gribben J, et al: Phase II study of single-agent bortezomib for the treatment of patients with fludarabine-refractory B-cell chronic lymphocytic leukemia. Cancer 107: 916-924, 2006.

18. Byrd JC, Marcucci G, Parthum MR, et al: A phase I and pharmacodynamic study of depsipeptide (FK220) in chronic lymphocytic leukemia and acute myeloid leukemia. Blood 105: 959-967, 2005.

19. Quiney C, Billard C, Faussat AM, et al: Pro-apoptotic properties of hyperforin in leukemic cells from patients with chronic lymphocytic leukemia. Leukemia 20: 491-497, 2006.

20. Quiney C, Billard C, Salanoubat C, Fourneron JD and Kolb JP: Hyperforin, a new lead compound against the progression of cancer and leukemia? Leukemia 20: 1519-1525, 2006.

21. Quiney C, Billard C, Mirshahi P, Fourneron JD and Kolb JP: Hyperforin inhibits MMP-9 secretion by B-CLL cells and microtubule formation by endothelial cells. Leukemia 20: 583-589, 2006.

22. Quiney C, Billard C, Faussat AM, Salanoubat C and Kolb JP: Hyperforin inhibits P-gp and BCRP activities in chronic lymphocytic leukaemia cells and myeloid cells. Leuk Lymphoma 48: 1587-1599, 2007

23. Zaher M, Akrout I, Mirshahi M, Kolb JP and Billard C: Noxa upregulation is associated with apoptosis of chronic lymphocytic leukemia cells induced by hyperforin but not flavopiridol. Leukemia 23: 594-596, 2009.

24. Hallek M, Cheson BD, Catovsky D, et al: Guidelines for the diagnosis and treatment of chronic lymphocytic leukemia: a report from the International Workshop on Chronic Lymphocytic Leukemia updating the National Cancer Institute-Working Group 1996 guidelines. Blood 111: 5446-5456, 2008.

25. Billard C, Kern C, Tang R, Ajchenbaum-Cymbalista F and Kolb JP: Flavopiridol downregulates the expression of both the inducible NO synthase and p2 $7^{\mathrm{kipl}}$ in malignant cells from B-cell chronic lymphocytic leukemia. Leukemia 17: 2345-2443, 2003.

26. Stacchini A, Aragno M, Vallario A, et al: MEC1 and MEC2: two new cell lines derived from B-chronic lymphocytic leukaemia in prolymphocytoid transformation. Leuk Res 23: 127-136, 1999.

27. Fourneron JD and Nait-Si Y: Effect of eluent $\mathrm{pH}$ on HPLC/UV analysis of hyperforin. Phytochem Anal 17: 71-77, 2006.

28. Moravec RA, O'Brien MA, Daily WJ, Scurria MA, Bernad L and Riss TL: Cell-based bioluminescent assays for all three proteasome activities in a homogeneous format. Anal Biochem 387: 294-302, 2009.

29. Kern C, Cornuel J, Billard C, et al: Involvement of BAFF and APRIL in the resistance to apoptosis of B-CLL through an autocrine pathway. Blood 103: 679-688, 2004.

30. Nencioni A, Grünebach F, Patrone F, Ballestrero A and Brossart P: Proteasome inhibitors: antitumor effects and beyond. Leukemia 21: 30-36, 2007.

31. Chen D, Daniel KG, Chen MS, Kuhn DJ, Landis-Piwowar KR and Ping Dou Q: Dietary flavonoid as proteasome inhibitors and apoptosis inducers in human leukemia cells. Biochem Pharmacol 69: 1421-1432, 2005. 
32. Dai Y, De Sano J, Tang W, et al: Natural proteasome inhibitor celastrol suppresses androgen-independent prostate cancer progression by modulating apoptotic proteins and NF-kappaB. PloS One 5: e14153, 2010

33. Baou M, Kohlhaas SL, Butterworth M, et al: Role of Noxa and its ubiquitination in proteasome inhibitor-induced apoptosis in chronic lymphocytic leukemia cells. Haematologica 95: 1510-1518, 2010.

34. Qin JZ, Ziffra J, Stennett L, et al: Proteasome inhibitors trigger Noxa-mediated apoptosis in melanoma and myeloma cells. Cancer Res 65: 6282-6293, 2005.

35. Li R, Zang Y,Li C, Patel NS, Grandis JR and Johnson DE: ABT-737 synergizes with chemotherapy to kill head and neck squamous cell carcinoma cells via a Noxa-mediated pathway. Mol Pharmacol 75 : 1231-1239, 2009

36. Ploner C, Kofler R and Villunger A: Noxa: at the tip of the balance between life and death. Oncogene 27: S84-S92, 2009.

37. Mackus WJM, Kater AP, Grummels A, et al: Chronic lymphocytic leukemia cells display p53-dependent drug-induced Puma upregulation. Leukemia 19: 427-434, 2005.

38. Iglesias-Serret D, De Frias M, Sanidrian AF, et al: Regulation of the proapoptotic BH3-only protein BIM by glucocorticoids, survival signals and proteasome in chronic lymphocytic leukemia cells. Leukemia 21: 281-287, 2007.

39. Ghiotto F, Fais F, Tenca C, et al: Apoptosis of B-cell chronic lymphocytic leukemia cells induced by a novel $\mathrm{BH} 3$ peptidomimetic. Cancer Biol Ther 8: 263-271, 2009.
40. Vogler M, Butterworth M, Majid A, et al: Concurrent up-regulation of BCL-XL and BCL2A1 induces approximately 1000-fold resistance to ABT-737 in chronic lymphocytic leukemia. Blood 113: 4403-441, 2009

41. Merle-Béral H, Barbier S, Roué G, Bras M, Sarfati M and Susin SA: Caspase-independent type III PCD: a new means to modulate cell death in chronic lymphocytic leukemia. Leukemia 23: 974-977, 2009.

42. Gey C, Kyrylenko S, Hennig L, Nguyen LHD, Büttner A, Pham HD and Giannis A: Phloroglucinol derivatives guttiferone $\mathrm{G}$, aristoforin, and hyperforin: inhibitors of human sirtuins SIRT1 and SIRT2. Angew Chem Int Ed 46: 5219-5222, 2007.

43. Rothley M, Schmid A, Thiele W, et al: Hyperforin and aristoforin inhibit lymphatic endothelial cell proliferation in vitro and in vivo and suppress tumor-induced lymphangiogenesis in vivo. Int J Cancer 125: 34-42, 2009.

44. O'Brien SM, Claxton DF, Crump M, et al: Phase I study of obatoclax mesylate (GX15-070), a small molecule pan-Bcl-2 family antagonist, in patients with advanced chronic lymphocytic leukemia. Blood 113: 299-305, 2009.

45. Balakrishnan K, Burger JA, Wierda WG and Gandhi V: AT-101 induces apoptosis in CLL B cells and overcomes stromal cellmediated Mcl-1 induction and drug resistance. Blood 113: 149-153, 2009. 